\title{
Dasatinib-Induced Hepatic Dysfunction
}

\author{
Arumugam Manoharan \\ Graduate School of Medicine, University of Wollongong, Wollongong, Australia. \\ Email: Prof_Manoharan@uow.edu.au
}

Received November 20 $0^{\text {th }}$ 2012; revised December 30 ${ }^{\text {th }}$, 2012; accepted January $19^{\text {th }}, 2013$

\begin{abstract}
A 53-year-old man with chronic myeloid leukaemia developed significant hepatic dysfunction when treatment was changed from imatinib (because of drug-induced rash) to dasatinib. Liver function tests returned to normal 77 days after cessation of therapy and have remained normal despite recommencement of dasatinib. Although the pathogenesis for the significant hepatic dysfunction is unclear, this case illustrates the reversibility of this event with dose interruption and that dasatinib can be safely recommenced for ongoing treatment.
\end{abstract}

Keywords: Dasatinib; Hepatic Dysfunction; Reversibility

\section{Introduction}

Dasatinib is one of the three tyrosine kinase inhibitor drugs, currently available to treat patients with chronic myeloid leukaemia (CML). It is used either as first line therapy or, more commonly, as second-line therapy in patients who are resistant to or intolerant of imatinib. [1] Hepatic dysfunction is a rare complication of dasatinib therapy, documented in less than $1 \%$ of patients; in these cases, the liver function abnormalities have been mild and generally resolved despite continuation of therapy. $[2,3]$ This report describes a patient with CML who developed significant hepatic dysfunction when treatment was changed from imatinib to dasatinib.

\section{Case Report}

In June, 2011, a 53 year old man was found to have leucocytosis and thrombocytosis on routine blood tests, suggestive of a myeloproliferative disorder. His past medical history only included childhood osteomyelitis. Physical examination showed a ptosed liver of $13 \mathrm{~cm}$ span and dullness over the splenic region. Blood counts showed haemoglobin $152 \mathrm{~g} / \mathrm{l}$, white cell count $19.4 \times 10^{\wedge} \mathrm{g} / \mathrm{l}$ (neutrophils 10.86, eosinophils 1.55, basophils 1.97, myelocytes 1.36) and platelets $500 \times 10^{\wedge} 9 / 1$. Bone marrow examination showed features of chronic myeloid leukaemia in chronic phase. Cytogenetic and molecular studies showed $\mathrm{Ph}_{1}$ chromosome and BCR-ABL translocation, confirming the diagnosis of CML.

He was commenced on imatinib $600 \mathrm{mg}$ daily. Two weeks later he presented with a generalised erythematous rash and some joint aches and pains. He responded well to prednisolone therapy $(25 \mathrm{mg} / \mathrm{d})$ but the rash recurred whenever the dosage was reduced to less than $20 \mathrm{mg} / \mathrm{d}$. Blood counts after four weeks of imatinib therapy showed haemoglobin $141 \mathrm{~g} / \mathrm{l}$, white cell count $5.9 \times$ 10^9/1 (normal differential) and platelets $195 \times 10^{\wedge} 9 / 1$. In December, 2011, he was switched over to dasatinib 100 mg daily. His rash resolved and one month later blood counts remained normal. However, his liver function tests (which had hitherto been normal) became significantly abnormal continued to rise over the next 10 days after cessation of dasatinib therapy (Table 1).

The following investigations were negative/normal: hepatitis A, B, C serology, Ross River virus serology, auto-immune serology including anti-mitochondrial antibody and anti-smooth muscle antibody and coagulation studies. Abdominal ultrasound examination only showed mild fatty infiltration of the liver.

He remained clinically well, as were his blood counts; his liver function tests gradually returned to normal (Table 1). In April, 2012 (77 days after cessation of therapy), dasatinib $100 \mathrm{mg}$ daily was recommenced. At the time of this report (November, 2012), he is well with normal liver function tests and blood counts. Peripheral blood quantitative RT PCR studies showed 0.26\% t (9:22) transcript, indicating optimal response to therapy.

\section{Discussion}

Significant hepatic dysfunction, as seen in this patient, is a hitherto unreported side-effect of dasatinib therapy. The patient was off imatinib and was on reducing doses of prednisolone therapy when the hepatic dysfunction occurred. The significant hepatic toxicity in this patient could only be attributed to dasatinib therapy. The pathogenesis for the significant but clinically asymptomatic 
Table 1. Results of liver function tests.

\begin{tabular}{|c|c|c|c|c|c|}
\hline Tests & Normal Range (u/l) & Pre-dasatinib therapy & On dasatinib 30 days & 10 days after cessation & 77 days after cessation \\
\hline Alkaline phosphatase & $35-100$ & 64 & 132 & 194 & 55 \\
\hline $\begin{array}{l}\text { Gamma glutamyl } \\
\text { transpeptidase }\end{array}$ & $5-50$ & 20 & 172 & 389 & 36 \\
\hline $\begin{array}{l}\text { Aspartate amino } \\
\text { transferase }\end{array}$ & $10-40$ & 18 & 453 & 673 & 25 \\
\hline $\begin{array}{l}\text { Alanine amino } \\
\text { transferase }\end{array}$ & $5-40$ & 19 & 977 & 1523 & 28 \\
\hline Lactic dehydrogenase & $120-250$ & 246 & 647 & 702 & 244 \\
\hline
\end{tabular}

hepatic dysfunction in our patient is unclear. As this adverse event has not recurred on re-challenge, one wonders whether it may be due to interaction with the previous tyrosine kinase inhibitor drug imatinib (which caused troublesome rash). Intolerance to second generation of tyrosine kinase inhibitors can sometimes result from cross intolerance with imatinib but the reports hitherto have only documented the re-emergence of the same adverse event that led to the discontinuation of imatinib [4].

Whatever the pathogenesis, this case illustrates the reversibility of hepatic toxicity with dose interruption and that dasatinib can be safely recommenced for ongoing treatment. This case also highlights the need for periodic monitoring of liver function tests when patients are commenced on dasatinib, particularly if they have pre-existing hepatic dysfunction or if they are on other drugs with hepatotoxic potential.

\section{REFERENCES}

[1] E. Jabbour and H. Kantarjian, "Chronic Myeloid Leukaemia: 2012 Update of Diagnosis, Monitoring and Management," American Journal of Hematology, Vol. 87, No. 11, 2012, pp. 1037- 1045. doi;10.1002/ajh.23282
[2] J. E. Cortes, D. Jones, S. O’Brien, E. Jabbour, F. Ravandi, C. Koller, et al., "Results of Dasatinib Therapy in Patients with Early Chronic-Phase Chronic Myeloid Leukemia," Journal of Clinical Oncology, Vol. 28, No. 3, 2010, 398404. doi;10.1200/JCO.2009.25.4920,

[3] "Bristol-Myers Squibb Company Sprycel ${ }^{\circledR}$ (Dasatinib) Product Information,” Bristol-Myers Squibb Australia Pty Ltd., Melbourne, 2011.

[4] E. Jabbour, M. Deininger and A. Hochhaus, "Management of Adverse Events Associated with Tyrosine Kinase Inhibitors in the Treatment of Chronic Myeloid Leukemia. Leukemia,” Vol. 25, No. 2, 2011, pp. 201-210. doi;10.1038/leu.2010.215 